\title{
Increase of Carrier-to-Noise Ratio in GPS Receivers Caused by Continuous-Wave Interference
}

\author{
Jian LI, Junwei NIE, Baiyu LI, Feixue WANG \\ College of Electronic Science and Engineering, National University of Defense Technology, 410073 Changsha, China \\ kleesword@163.com,wangfeixue_nnc@163.com \\ Manuscript received September 16, 2015
}

\begin{abstract}
The increased use of personal private devices (PPDs) is drawing greater attention to the effects of continuous-wave interference (CWI) on the performance of global positioning system (GPS) receivers. The effective carrier-to-noise density ratio $\left(C / N_{0}\right)$, an essential index of GNSS receiver performance, is studied in this paper.

Receiver tracking performance deteriorates in the presence of interference. Hence, the effective $C / N_{0}$, which measures tracking performance, decreases. However, simulations and bench tests have shown that the effective $C / N_{0}$ may increase in the presence of $C W I$. The reason is that a sinusoidal signal is induced by the CWI in the correlator and may be tracked by the carrier tracking loop. Thus, the effective carrier power depends on the power of the signal induced by the CWI, and the effective $C / N_{0}$ increases with the power of the CWI. The filtering of the CWI in the carrier tracking loop correlator and its effect on the phase locked loop (PLL) tracking performance are analyzed. A mathematical model of the effect of the CWI on the effective $C / N_{0}$ is derived. Simulation results show that the proposed model is more accurate than existing models, especially when the jam-to-signal ratio (JSR) is greater than $30 \mathrm{dBc}$.
\end{abstract}

\section{Keywords}

Receiver performance, interference monitoring, signal-to-interference-plus-noise ratio

\section{Introduction}

In global position system (GPS), understanding the effects of interference is important for conducting threat assessments, mitigation research and selecting ground control sites. Thus, it is necessary to establish accurate models of the effects of interference on a receiver.

Assessments of the effects of interference usually focus on indexes such as the post-correlation effective carrier-to-noise density ratio $\left(C / N_{0}\right)$ and the code tracking error [1-4]. The effective $C / N_{0}$ has been investigated in many studies because it is used in assessing interference effects on acquisition, carrier tracking and data demodulation in receivers [5-7]. Early research by Betz used estimation theory with the assumptions of partial-band interference and Gaussian distributions to evaluate the effective $C / N_{0}$ of receivers [1]. Bek et al. conducted mathematical analyses of pulse interference on the effective $C / N_{0}$ in GPS receivers [8]. Research on mitigation techniques for GNSS receivers has drawn increasing attention [9-11]. Omar derived measures of the code tracking error in receivers using time-domain blanking and frequency-domain adaptive filtering [9].

These studies on interference concentrated on complex modulation interference and mitigation. Another topic of research is the effect of CWI on the effective $C / N_{0}$ [12-15]. The increased use of personal private devices (PPDs) [16], [17] has generated greater interest in the effects of CWI on civilian receivers. However, the effective $C / N_{0}$ models developed for partial-band interference in [1] cannot be applied to CWI because bandwidth of CWI is too narrow to use continuous spectral density of navigation signal [14]. The spectrum of navigation signals should be modeled as a line spectrum in the presence of CWI because of the pseudo-random noise (PRN) code repetition [15]. An important conclusion in [15] was that CWI induces the maximum loss in the effective $C / N_{0}$ when the frequency of the CWI coincides with the worst spectral line of the navigation signal. In [13-15], wrongly applying continuous power spectrum density in [1] to continuous-wave Dirac delta function is removed. However, the effect of the CWI on the PLL tracking was neglected. If the power of a sinusoidal signal or a constant term resulting from CWI passing through the correlator is greater than that of the navigation signal, the PLL may track the signal resulting from the CWI. This behavior may cause the effective $C / N_{0}$ to increase with the jam-to-signal ratio (JSR), which has been observed in simulations and lab tests of interference modulation schemes, but this is inconsistent with the effective $C / N_{0}$ estimation model presented in [15].

The goal of this study was to clarify the effects of CWI on the effective $C / N_{0}$ of GPS receivers and other GNSS receivers using binary phase-shift keying codes. The contributions of this study can be summarized as follows:

A. The filtering of the CWI in the correlator of the 
carrier tracking loop is detailed.

B. A more accurate mathematical model for assessing the effect of CWI on the effective $C / N_{0}$ of GPS receivers is developed.

This paper is organized as follows. In Sec. 2, the carrier tracking loop and the intermediate-frequency signal are briefly discussed. In Sec. 3, the filtering of the CWI in the correlator is examined, and its effect on the carrier tracking loop is analyzed. In Sec. 4, a model of the effect of CWI on the effective $C / N_{0}$ is derived. In Sec. 5 , results of simulations conducted to validate the proposed effective $C / N_{0}$ model are presented. Section 6 presents conclusions drawn from this research.

\section{Receiver Carrier Tracking Loop}

The carrier tracking loop of a GPS receiver is shown in Fig. 1.

The carrier tracking loop shown in Fig. 1 is essentially a phase-locked loop (PLL). A Costas PLL is used in most GPS receivers. The intermediate-frequency input signal $x(t)$ is defined as follows:

$$
\begin{aligned}
x(t) & =s(t)+l(t)+n(t) \\
& =a_{\mathrm{s}} d(t) c\left(t-\tau_{\mathrm{s}}\right) \exp \left\{j\left[2 \pi\left(f_{\mathrm{c}}+f_{\mathrm{d}}\right) t+\theta_{\mathrm{s}}\right]\right\} \\
& +a_{1} \exp \left[j\left(2 \pi f_{\mathrm{l}} t+\theta_{1}\right)\right]+n(t)
\end{aligned}
$$

where $a_{\mathrm{s}}$ is the amplitude of the navigation signal $s(t)$, $c\left(t-\tau_{\mathrm{s}}\right)$ is the PRN code, $\tau_{\mathrm{s}}$ is the time of arrival of the signal, $d(t)$ is the navigation data, $f_{\mathrm{c}}$ is the carrier frequency, $f_{\mathrm{d}}$ is the Doppler frequency, $\theta_{\mathrm{s}}$ is the initial phase of the carrier, $f_{1}$ is the frequency of the interference $l(t), \theta_{1}$ is the initial phase of $l(t)$, and $n(t)$ is Gaussian white noise; i.e., $n(t) \propto N\left(0, \sigma^{2}\right)$. The baseband signals $i$ and $q$ are acquired by removing the carrier signal and the PRN code from $x(t)$. Signals $i$ and $q$ pass through an integrate-dump filter to generate $I$ and $Q$, respectively, as shown in Fig. 1 . These are the main functions of a correlator. The carrier numerically controlled oscillator (NCO) is time adjusted to guarantee that the output of the phase discriminator is zero.

The code-tracking loop (or delay-locked loop (DLL)) of a GPS receiver performs either coherent or non-coherent early-late processing (NELP). Correspondingly, the postcorrelation signal-to-interference-plus-noise ratio (SINR) can be defined as either the coherent or non-coherent

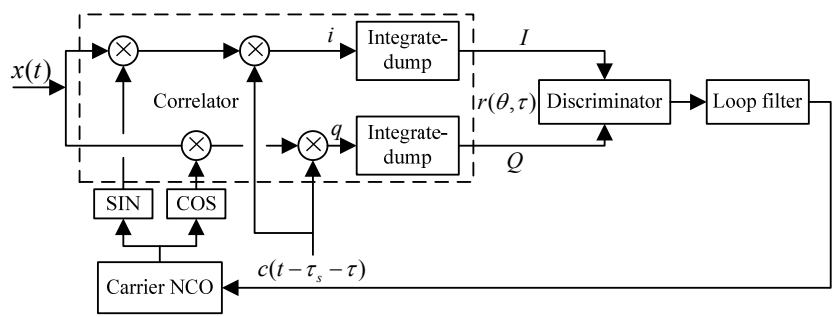

Fig. 1. GPS receiver carrier tracking loop.
SINR. The effective $C / N_{0}$ is sufficient for characterizing both the coherent and non-coherent SINRs [1]. In the present study, the coherent SINR is selected to derive the effective $C / N_{0}$. The coherent $S I N R$ is defined as follows:

$$
\operatorname{SINR}=\frac{|E\{I\}|^{2}}{\operatorname{var}\{I\}}
$$

where $E\{\cdot\}$ denotes the expectation operator and $\operatorname{var}\{\cdot\}$ denotes the variance.

The relation between the coherent SINR and the effective $C / N_{0}$ is as follows [1]:

$$
C N_{0 \mathrm{eff}}=\frac{S I N R}{2 T \int_{-B / 2}^{B / 2} G_{\mathrm{s}}(f) \mathrm{d} f}
$$

where $T$ is the integration interval, $B$ is the front-end band and $G_{\mathrm{s}}(f)$ is the normalized power spectral density of the navigation signal.

The signal at the output of integrate-dump filter is

$$
\begin{aligned}
r(\theta, \tau)= & \frac{1}{T} \int_{-T / 2}^{T / 2} x(t) s_{0}^{*}(t-\tau) \exp (j \theta) \mathrm{d} t \\
= & \frac{1}{T} \int_{-T / 2}^{T / 2} s(t) s_{0}^{*}(t-\tau) \exp (j \theta) \mathrm{d} t \\
& +\frac{1}{T} \int_{-T / 2}^{T / 2} l(t) s_{0}^{*}(t-\tau) \exp (j \theta) \mathrm{d} t \\
& +\frac{1}{T} \int_{-T / 2}^{T / 2} n(t) s_{0}^{*}(t-\tau) \exp (j \theta) \mathrm{d} t \\
= & r_{\mathrm{ss}}(\theta, \tau)+r_{\mathrm{ls}}(\theta, \tau)+r_{\mathrm{ws}}(\theta, \tau)
\end{aligned}
$$

where $\theta$ is the carrier tacking phase error, $\tau$ is the tracking code delay, $s_{0}(t)$ is the reference signal and $s(t)$ approximates the normal navigation signal.

\section{Effect of CWI on the Carrier Tracking Loop}

\subsection{Filtering of the CWI in the Correlator}

The transformation of the CWI signal as it passes from the intermediate-frequency input to the integratedump filter output is analyzed on the premise that the reference signal $s_{0}(t)$ is in phase with the carrier signal and the PRN code delay is zero; that is, $\theta=0, \tau=0$. Thus, the output of the integrate-dump filter induced by the CWI is given by (5),

$$
\begin{aligned}
& r_{\mathrm{ls}}(\theta, \tau, U)=\frac{1}{T} \int_{-T / 2}^{T / 2} \exp (j \theta) l(t) s_{0}^{*}(t-\tau) \mathrm{d} t \\
& =\frac{a_{l}}{T} \int_{-T / 2}^{T / 2} \exp \left[j\left(2 \pi f_{\Delta} t+\theta+\theta_{1}-\theta_{\mathrm{s}}\right)\right] c\left(t-\tau_{\mathrm{s}}-\tau\right) \mathrm{d} t \\
& =\frac{a_{l}}{T} \int_{-\frac{T}{2}-\tau}^{\frac{T}{2}-\tau} \exp \left[j\left(2 \pi f_{\Delta} u+\beta\right)\right] c\left(u-\tau_{\mathrm{s}}\right) \mathrm{d} u \\
& \approx a_{1} \exp (j \beta) S\left(-f_{\Delta}\right)=a_{1}\left|S\left(f_{\Delta}\right)\right| \exp \left[j\left(2 \pi f_{\mathrm{rem}} U+\beta-\phi\right)\right]
\end{aligned}
$$


where $U$ is the sample sequence number following the integrate-dump operation, $\beta=2 \pi f_{\Delta} \tau+\theta+\theta_{l}-\theta_{s}$, $|\cdot|$ denotes absolute value, $f_{\Delta}=f_{1}-f_{\mathrm{c}}-f_{\mathrm{d}}$ is the difference between the carrier and CWI frequencies, $F_{\mathrm{c}}=1 / T_{\mathrm{c}}$ is the PRN code repetition frequency, and $T_{\mathrm{c}}$ is the PRN code repetition interval. The frequency $f_{\text {rem }}$ of the signal output by the correlator is

$$
f_{\text {rem }}= \begin{cases}\bmod \left(f_{\Delta}, F_{\mathrm{c}}\right), & \text { if } \bmod \left(f_{\Delta}, F_{\mathrm{c}}\right)<F_{\mathrm{c}} / 2 \\ -\bmod \left(f_{\Delta}, F_{\mathrm{c}}\right), & \text { if } \bmod \left(f_{\Delta}, F_{\mathrm{c}}\right) \geq F_{\mathrm{c}} / 2\end{cases}
$$

The phase of $S\left(f_{\Delta}\right)$ is

$$
\phi=\tan ^{-1}\left(\frac{\operatorname{Im}\left\{S\left(f_{\Delta}\right)\right\}}{\operatorname{Re}\left\{S\left(f_{\Delta}\right)\right\}}\right)
$$

where the normal spectrum of the navigation signal is defined as

$$
|S(f)|=\left|\frac{1}{T} \int_{-T / 2}^{T / 2} c\left(t-\tau_{\mathrm{s}}\right) \exp (-j 2 \pi f t) \mathrm{d} t\right| .
$$

The navigation signal has a line spectrum, and the interval between the spectral lines is $F_{\mathrm{c}}$ [15]. The in-phase and quadrature components of the output of the integratedump filter are expressed as follows:

$$
\begin{aligned}
I_{\mathrm{ls}} & =\operatorname{Re}\left\{r_{\mathrm{ls}}(t)\right\} \\
& =a_{1}\left|S\left(f_{\Delta}\right)\right| \cos \left(2 \pi f_{\mathrm{rem}} U+\beta-\phi\right), \\
Q_{\mathrm{ls}} & =\operatorname{Im}\left\{r_{\mathrm{ls}}(t)\right\} \\
& =a_{1}\left|S\left(f_{\Delta}\right)\right| \sin \left(2 \pi f_{\mathrm{rem}} U+\beta-\phi\right) .
\end{aligned}
$$

Equations (9) and (10) show that the frequency of the filtered CWI signal output by the correlator is $f_{\text {rem. The }}$ amplitude of this signal depends on the amplitude of the CWI at the intermediate-frequency input and the normal navigation signal spectral value at $f_{\Delta}$. Alternatively, the product of the PRN code $c\left(u-\tau_{\mathrm{s}}\right)$ and $\exp \left(j 2 \pi f_{\Delta} u\right)$ in the time domain in (5) shifts the frequency of the PRN code up by $f_{\Delta} \mathrm{Hz}$. The integration operation $\int_{-T / 2}^{T / 2} \cdot \mathrm{d} t$ can be regarded as low-pass filtering with the cut-off frequency $f_{\Delta}=1 / T \mathrm{~Hz}$. If $f_{\Delta} \neq l F_{c} \mathrm{~Hz},(l=0, \pm 1, \pm 2, \ldots)$, the spectral line at $-f_{\Delta} \mathrm{Hz}$ is shifted to a frequency between zero and $f_{\text {cut }}$. Usually, $T \geq T_{\mathrm{c}}$, so $f_{\text {cut }} \leq F_{\mathrm{c}}$. Because of the low pass filter with the cut-off frequency $f_{\text {cut }} \mathrm{Hz}$, only one spectral line remains. Thus, the CWI generates an output from the correlator with a frequency of $f_{\text {rem }}=\bmod \left(f_{\Delta}, F_{c}\right)$. The case where $f_{\text {rem }}=0$, namely, where the frequency of the CWI coincides with that of the navigation signal spectral lines, will not be analyzed here; this case will be covered in future research.

The following example illustrates the aforementioned process. It is assumed that the power of the CWI is unity, the sequence number of the coarse acquisition (C/A) PRN code is 6 (i.e., PRN \#6), $T$ is $1 \mathrm{~ms}$ or $10 \mathrm{~ms}, f_{\Delta}$ is $-226.95 \mathrm{kHz}, \tau=0$ and $\beta=0$. From (9) and (10), $I_{\mathrm{ls}}=a_{1}\left|S\left(f_{\Delta}\right)\right| \cos \left(2 \pi f_{\text {rem }} U\right)$ and $Q_{\mathrm{ls}}=a_{1}\left|S\left(f_{\Delta}\right)\right| \sin \left(2 \pi f_{\text {rem }} U\right)$. The signal in (5) is shown in the frequency domain in Fig. 2, where the horizontal axis is frequency and the vertical axis is amplitude.

The PRN code spectrum in the left subfigure of Fig. 2 is shifted down to $226.95 \mathrm{kHz}$. In other words, the spectral line at $227 \mathrm{kHz}$ is shifted left by $50 \mathrm{~Hz}$. The shifted line spectrum passes through a low-pass filter (shown by the solid line in the right subfigure) and remains sinusoidal with a frequency of $50 \mathrm{~Hz}$. The local spectrum of the PRN code is shown for the case $T=1 \mathrm{~ms}$.

The real and imaginary parts and the envelope of the spectrum are shown in Fig. 3, where the horizontal axis is frequency and the vertical axis is amplitude. The spectrum envelope is the same as that in left subfigure of Fig. 2 for the case $T=1 \mathrm{~ms}$. The components $I_{\mathrm{ls}}$ and $Q_{\mathrm{ls}}$ are shown in Fig. 4.

Figure 4 shows $I_{\mathrm{ls}}, Q_{\mathrm{ls}}$ and the envelope of $r_{\mathrm{ls}}$, where the horizontal axis is time and the vertical axis is amplitude. The data are shown for the first $1 \mathrm{~ms}$. The results in Figs. 3 and 4 validate equations (9) and (10). The spectrum of $r_{\mathrm{ls}}$ is given in Fig. 5, which shows that the frequency of $r_{\mathrm{ls}}$ is approximately $50 \mathrm{~Hz}$.

In summary, CWI filtered by the correlator remains sinusoidal (if its frequency does not coincide with the spectral lines of the navigation signal, i.e., $\left.f_{\Delta} \neq l F_{c}\right)$. This conclusion is reached on the premise that the carrier and PRN code signals are in phase. If the PRN code signal is in
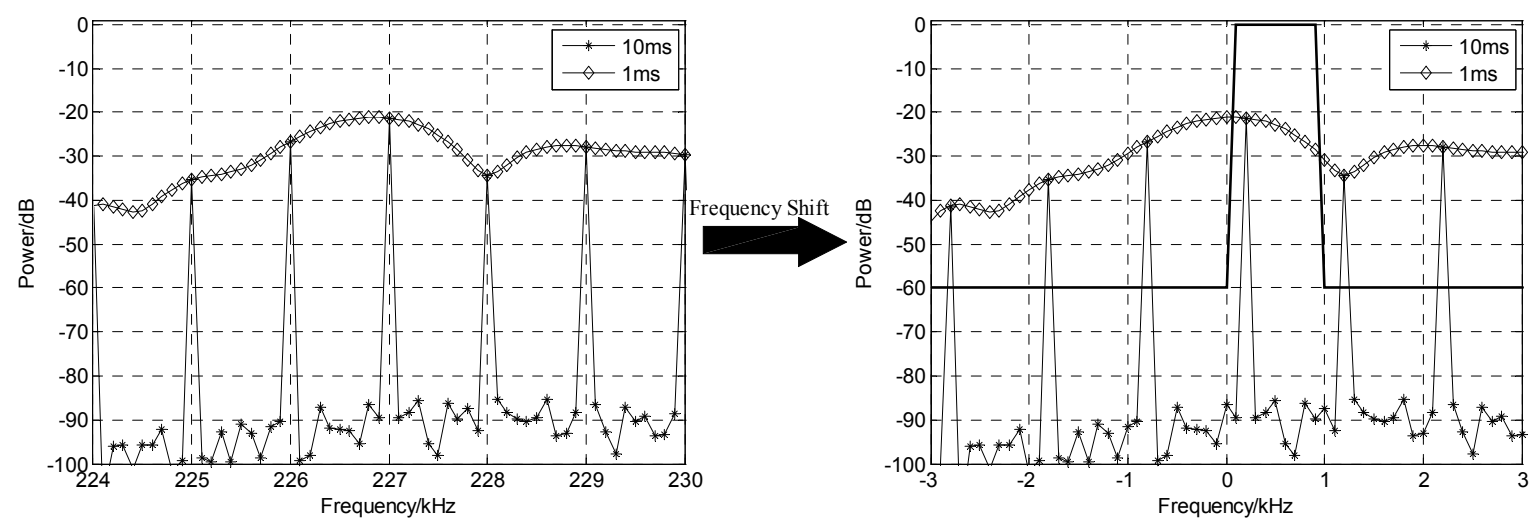

Fig. 2. Spectrum shifting and low-pass filtering. 


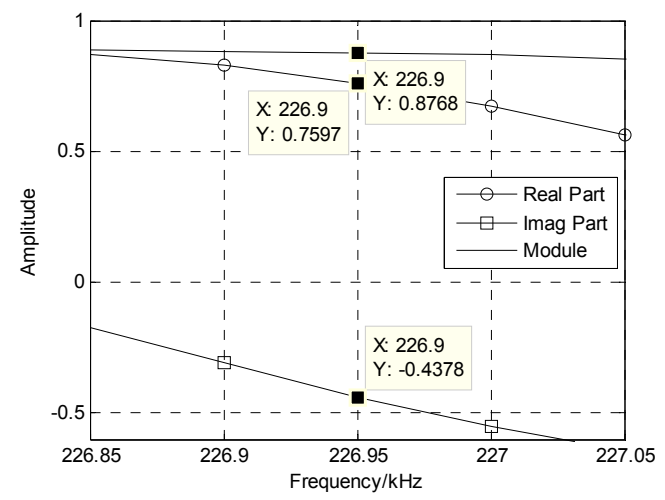

Fig. 3. Local spectrum of PRN \#6.

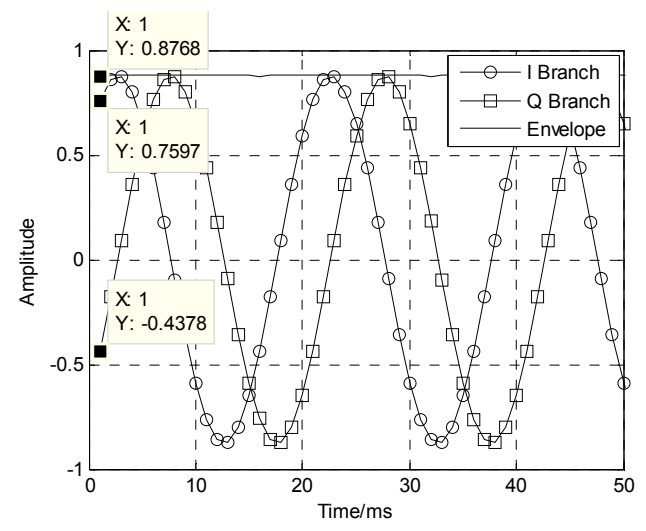

Fig. 4. Integrate-dump filter output.

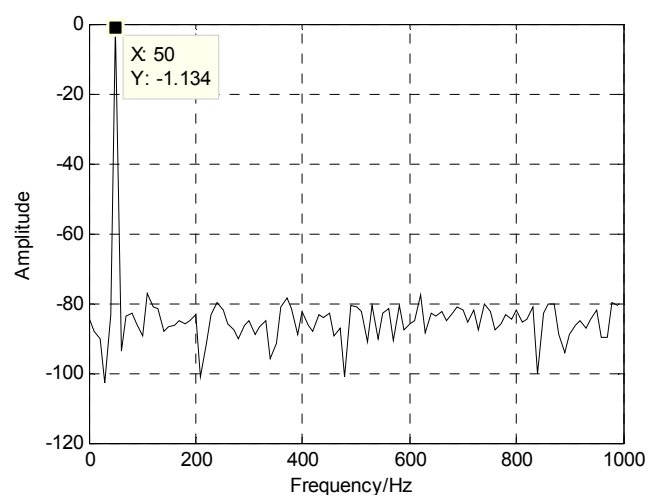

Fig. 5. Spectrum of $I_{\mathrm{s}}$.

phase, the navigation signal filtered by the correlator may also be a sinusoid. Because the filter outputs generated by the CWI and the navigation signal are fed into the tracking loop, either of them may be tracked by the PLL. Which signal is tracked depends on its power at the output of the correlator; i.e., the signal with the higher power will be tracked. The effect of CWI on the PLL tracking will be analyzed for various values of JSR.

\subsection{Effect of CWI on PLL Tracking}

We begin by examining the effect of CWI on a DLL. The NELP scheme is used in most receivers because of its insensitivity to carrier frequency errors and phase errors. The early and late components of a signal at the output of a DLL code correlator are as follows [3]:

$$
\begin{aligned}
E & =\left|r\left(\theta, \tau-\frac{\Delta}{2}\right)\right|^{2} \\
& =\left|r_{\mathrm{ss}}\left(\theta, \tau-\frac{\Delta}{2}\right)+r_{\mathrm{ls}}\left(\theta, \tau-\frac{\Delta}{2}\right)+r_{\mathrm{ws}}\left(\theta, \tau-\frac{\Delta}{2}\right)\right|^{2}, \\
L & =\left|r\left(\theta, \tau+\frac{\Delta}{2}\right)\right|^{2} \\
& =\left|r_{\mathrm{ss}}\left(\theta, \tau+\frac{\Delta}{2}\right)+r_{\mathrm{ls}}\left(\theta, \tau+\frac{\Delta}{2}\right)+r_{\mathrm{ws}}\left(\theta, \tau+\frac{\Delta}{2}\right)\right|^{2} .
\end{aligned}
$$

The error signal at the output of the code correlator is $E\{E-L\}=a_{\mathrm{s}}^{2} R_{\Delta / 2}^{2}-a_{\mathrm{s}}^{2} R_{-\Delta / 2}^{2}$ or $E\{E-L\}=0$ (see Appendix A for further details), where $R(\cdot)$ is the normal PRN code autocorrelation, $R_{\Delta}=R(\tau-\Delta)$, and $\Delta$ is the early-late spacing. Thus, $\tau=0$ in (4) when the PLL is in a locked condition, so CWI will not result in code tracking errors. Let the carrier tracking phase error be denoted by $\theta_{\delta}$. Then, the signal at the output of integrate-dump filter in Fig. 1 is

$$
r\left(\theta_{\delta}\right)=r_{\mathrm{ss}}\left(\theta_{\delta}\right)+r_{\mathrm{ls}}\left(\theta_{\delta}\right)+r_{\mathrm{ws}}\left(\theta_{\delta}\right) .
$$

The signal $r\left(\theta_{\delta}\right)$ in (13) is necessary to compute the effective $C / N_{0}$. In the previous section, it was shown that signals output by the correlator resulting from both CWI and the navigation signal enter the PLL, and the output with the higher power will be tracked. Depending on which signal the PLL tracks, the effective $C / N_{0}$ model may be different. Thus, in analyzing the effect of CWI on the PLL tracking, three values of the correlator JSR are considered. We define the correlator JSR as $\eta=a_{1}^{2}\left|S\left(f_{\Delta}\right)\right|^{2} / a_{\mathrm{s}}^{2}$.

The case without interference serves as the reference. Without interference, equation (13) can be written as $r\left(\theta_{\delta}\right)=r_{\mathrm{ss}}\left(\theta_{\delta}\right)+r_{\mathrm{ws}}\left(\theta_{\delta}\right)$, where $T$ is $1 \mathrm{~ms}$, the carrier-tonoise ratio (CNR) is $50 \mathrm{dBHz}$, and the other parameter values are as shown in Fig. 2. When the PLL is locked, the $Q$ component is noise and the $I$ component is a mix of a constant term from the signal and noise, as shown in Fig. 6, where the horizontal axis is time and the vertical axis is amplitude. The variation in the $I$ component is caused by the change in sign of the navigation signal. In the following, the effect of CWI on the PLL tracking will be discussed for three cases of correlator JSR $\eta$.

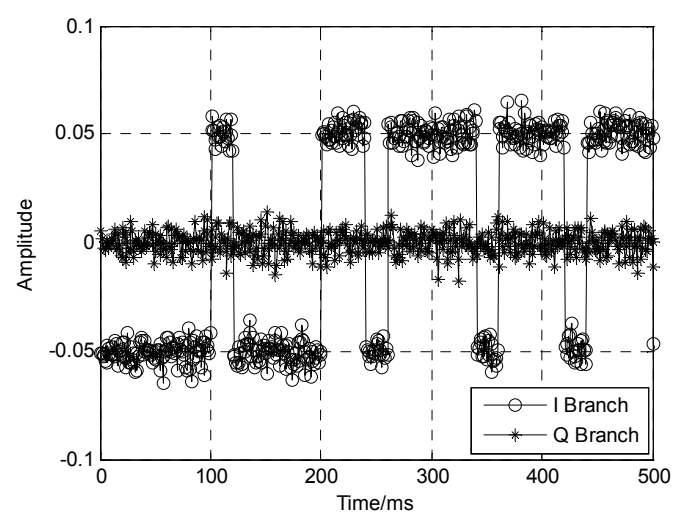

Fig. 6. Integrate-dump filter output (without jamming). 


\section{Case a: $\quad \eta<<1$}

In this case, the power of the filtered CWI signal (from the correlator) is less than that of the filtered navigation signal, so the PLL tracks the navigation signal. Substituting $\tau=0$ and $\Delta=0$ into (32) and (34) in Appendix A yields

$$
\begin{aligned}
& r\left(\theta_{\delta}\right) \\
& =r_{\mathrm{ss}}\left(\theta_{\delta}\right)+r_{\mathrm{ls}}\left(\theta_{\delta}\right)+r_{\mathrm{ws}}\left(\theta_{\delta}\right) \\
& =r_{\mathrm{ws}}\left(\theta_{\delta}\right)+d(T) a_{\mathrm{s}} \exp \left(j \theta_{\delta}\right) \\
& +a_{1}\left|S\left(f_{\Delta}\right)\right| \exp \left[j\left(2 \pi f_{\mathrm{rem}} U-\phi+\beta\right)\right] \\
& =r_{\mathrm{ws}}\left(\theta_{\delta}\right)+d(T) a_{\mathrm{s}} \\
& +a_{1}\left|S\left(f_{\Delta}\right)\right| \exp \left[j\left(2 \pi f_{\mathrm{rem}} U+\theta_{e}-\phi\right)\right]
\end{aligned}
$$

where $d(T)$ is the sign of the navigation signal.

Equation (14) shows that the $I$ component consists of noise, the filtered CWI signal and the constant term from the navigation signal. The JSR in this case is $10 \mathrm{dBc}$. The carrier tracking results are shown in Figs. 7 and 8 (the latter shows only the first $100 \mathrm{~ms}$ of the data). Comparing Figs. 6 and 7, it can be concluded that the amplitude of the I component is approximately equal in both. The amplitude of the $I$ component depends on the amplitude of the navigation signal, as indicated in (14). Figure 8 indicates that the sinusoidal signal exists in both the $I$ component and the $Q$ component. The frequency of the sinusoid is approximately $50 \mathrm{~Hz}$, which can be computed from $f_{\text {rem }}=$ $\bmod \left(-226.95 \times 10^{3}, 10^{3}\right)=50 \mathrm{~Hz}$.

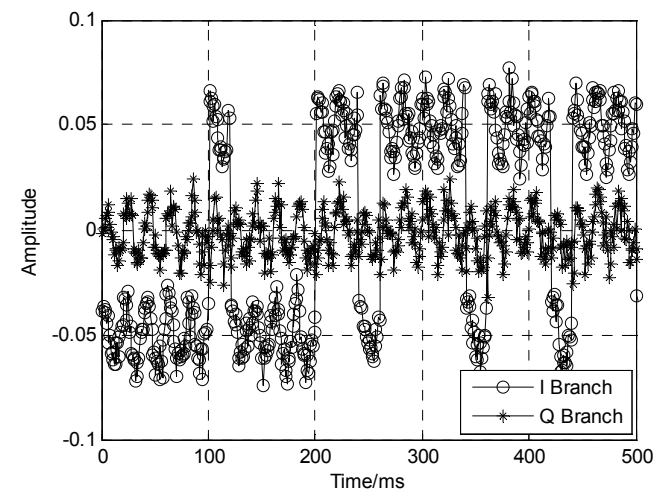

Fig. 7. Integrate-dump filter output $(J S R=10 \mathrm{dBc})$.

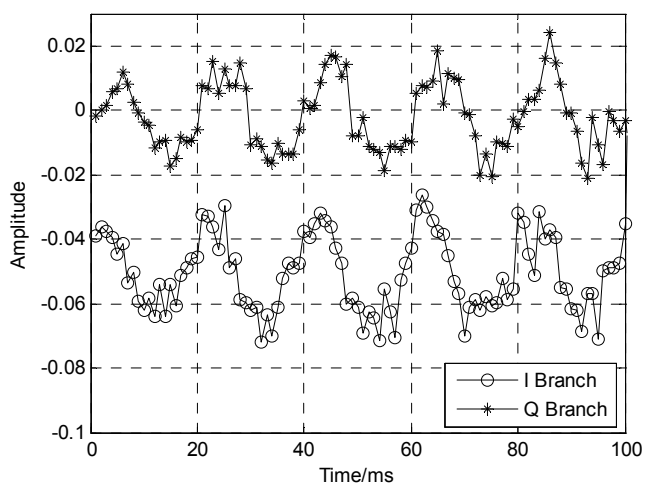

Fig. 8. Integrate-dump filter output for the first $100 \mathrm{~ms}$ $(J S R=10 \mathrm{dBc})$.
Case b: $\quad \eta>1$

In this case, the power of the filtered CWI signal is larger than that of the filtered navigation signal, so the PLL tracks the filtered CWI signal. Substituting $\tau=0$ and $\Delta=0$ into (40) and (42) in Appendix A yields

$$
\begin{aligned}
& r\left(\theta_{\delta}\right) \\
& =r_{\mathrm{ss}}\left(\theta_{\delta}\right)+r_{\mathrm{ls}}\left(\theta_{\delta}\right)+r_{\mathrm{ws}}\left(\theta_{\delta}\right) \\
& =r_{\mathrm{wt}}\left(\theta_{\delta}\right) \\
& +d(T) a_{\mathrm{s}} \exp \left[j\left(2 \pi f_{\mathrm{rem}} U+\theta-\theta_{\mathrm{e}}\right)\right] \\
& +a_{l}\left|S\left(f_{\Delta}-f_{\mathrm{rem}}\right)\right| \exp \left[j\left(\theta+\theta_{l}-\theta_{\mathrm{s}}-\phi\right)\right] \\
& =r_{\mathrm{wt}}\left(\theta_{\delta}\right)+a_{1}\left|S\left(f_{\Delta}-f_{\mathrm{rem}}\right)\right| \\
& +d(T) a_{\mathrm{s}} \exp \left[j\left(2 \pi f_{\mathrm{rem}} U+\phi-2 \theta_{\mathrm{e}}\right)\right] .
\end{aligned}
$$

Equation (15) shows that the $I$ component consists of noise, the filtered navigation signal and a constant term from the CWI. In this case, the JSR is $50 \mathrm{dBc}$. The carrier tracking results are shown in Fig. 9.

Comparing Figs. 6 and 9, it can be observed that the amplitude of the $I$ component is much higher in the latter, although the CNR is equal. The increase in the amplitude of the $I$ component results from the increase in power of the CWI, as (15) indicates. Figure 10 indicates that the sinusoidal signal exists in both the I component and the Q component. The frequency of this signal is approximately $50 \mathrm{~Hz}$, which can be computed from $f_{\text {rem }}=$ $\bmod \left(-226.95 \times 10^{3}, 10^{3}\right)=50 \mathrm{~Hz}$.

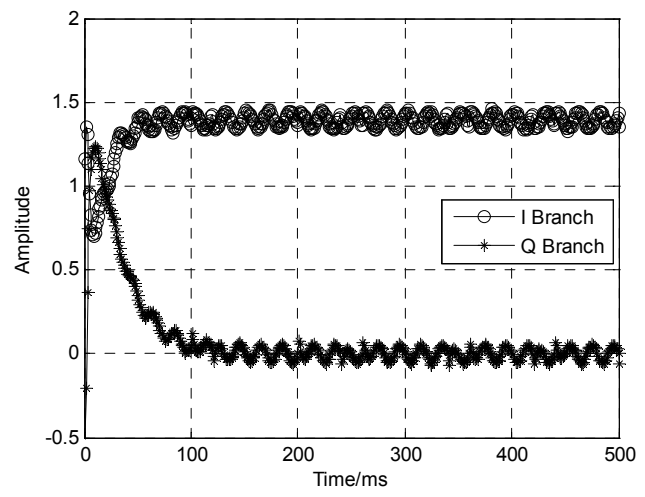

Fig. 9. Integrate-dump filter output $(J S R=50 \mathrm{dBc})$.

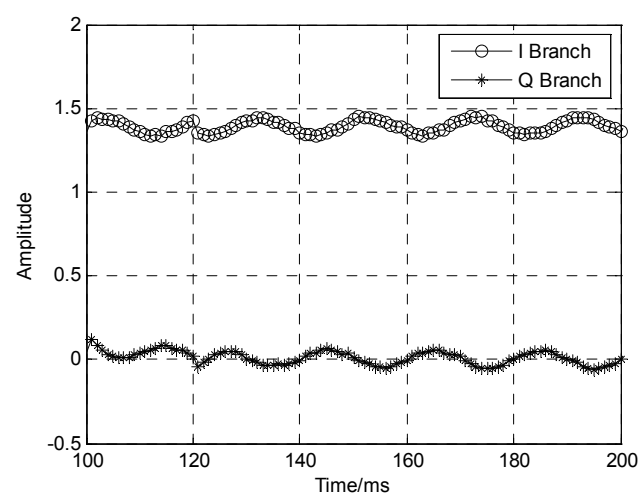

Fig. 10. Integrate-dump filter output for the first $200 \mathrm{~ms}$ $(J S R=50 \mathrm{dBc})$. 


\section{Case c: $\quad \eta \approx 1$}

In this case, the power of the filtered CWI signal is approximately equal to that of the filtered navigation signal, so the PLL may track the wrong signal or not track either signal. The JSR in this case is $20 \mathrm{dBc}$. The normal power of the strongest spectral line of the PRN 6 code is approximately $-21 \mathrm{~dB}$. Thus, $a_{1}\left|S\left(f_{\Delta}\right)\right|$ is approximately equal to $a_{\mathrm{s}}$. The carrier tracking results are shown in Fig. 11 which indicates that the PLL does not track either of the filtered CWI or navigation signals.

In summary, the effect of CWI on the PLL performance depends on the correlator JSR. The effect of CWI on the effective $C / N_{0}$ is analyzed in the following section.

\section{Effective $C / N_{0}$ Model}

The effect of CWI on the PLL performance depends on the JSR. Therefore, the effect of CWI on the effective $C / N_{0}$ depends on the JSR.

\section{Case a: $\quad \eta<<1$}

In this case, the carrier tracking loop tracks the navigation signal. The SINR will be used for this analysis. Firstly, $E\{I\}$ is analyzed according to (2), where $E\{I\}$ is derived from (14):

$$
E\{I\}=E\left\{\operatorname{Re}\left[r\left(\theta_{\delta}\right)\right]\right\}=d(T) a_{\mathrm{s}} .
$$

To compute the variance, the mean square value of the $I$ component is computed:

$$
\begin{aligned}
& E\left\{|I|^{2}\right\} \\
= & E\left\{\left|\operatorname{Re}\left\{r\left(\theta_{\delta}\right)\right\}\right|^{2}\right\} \\
= & E\left\{\left|\operatorname{Re}\left\{r_{\mathrm{ws}}\left(\theta_{\delta}\right)\right\}+d(T) a_{\mathrm{s}}+a_{1}\right| S\left(f_{\Delta}\right)\left|\cos \left(2 \pi f_{\text {rem }} U+\theta_{\mathrm{e}}-\phi\right)\right|^{2}\right\} \\
= & a_{\mathrm{s}}^{2}+\frac{1}{2} a_{1}^{2}\left|S\left(f_{\Delta}\right)\right|^{2}+\left|\operatorname{Re}\left\{r_{\mathrm{wt}}\left(\theta_{\delta}\right)\right\}\right|^{2} \\
= & a_{\mathrm{s}}^{2}+\frac{a_{1}^{2}\left|S\left(f_{\Delta}\right)\right|^{2}}{2}+\frac{1}{2 T} \int_{-B / 2}^{B / 2} G_{\mathrm{w}}(f)|S(f)|^{2} \mathrm{~d} f \\
= & |E\{I\}|^{2}+\frac{1}{2} a_{1}^{2}\left|S\left(f_{\Delta}\right)\right|^{2}+\frac{N_{0}}{2 T} \int_{-B / 2}^{B / 2} G_{\mathrm{s}}(f) \mathrm{d} f
\end{aligned}
$$

where $G_{\mathrm{s}}(f)$ is the normal power spectrum density of the navigation signal, $G_{\mathrm{w}}(f)$ is the noise power spectral density function and $B$ is the receiver band. The variance is

$$
\begin{aligned}
& \operatorname{Var}\{I(t)\} \\
& =E\left\{|I(t)|^{2}\right\}-|E\{I(t)\}|^{2} \\
& =\frac{N_{0}}{2 T} \int_{-B / 2}^{B / 2} G_{\mathrm{s}}(f) \mathrm{d} f+\frac{1}{2 T} \int_{-B / 2}^{B / 2} G_{1}(f) G_{\mathrm{s}}(f) \mathrm{d} f \\
& \approx \frac{N_{0}}{2 T}+\frac{a_{1}^{2}\left|S\left(f_{\Delta}\right)\right|^{2}}{2}
\end{aligned}
$$

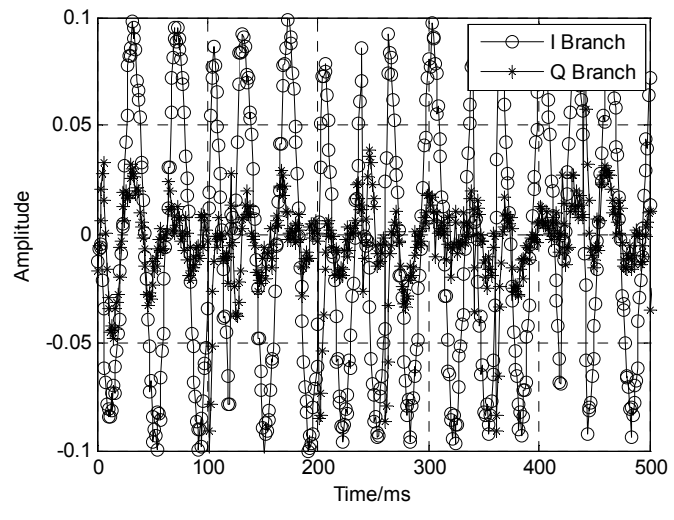

Fig. 11. Integrate-dump filter output $(J S R=20 \mathrm{dBc})$.

where $N_{0}$ is the noise power spectral density. Substituting (2), (16) and (18) into (3) yields

$$
\begin{aligned}
& C N 0_{\text {eff }} \\
= & \frac{|E\{I\}|^{2}}{\operatorname{var}\{I\}} \cdot \frac{1}{2 T \int_{-B / 2}^{B / 2} G_{\mathrm{s}}(f) \mathrm{d} f} \\
= & \frac{a_{s}^{2}}{N_{0}\left(\int_{-B / 2}^{B / 2} G_{\mathrm{s}}(f) \mathrm{d} f\right)^{2}+T a_{1}^{2}\left|S\left(f_{\Delta}\right)\right|^{2}} .
\end{aligned}
$$

Usually, the main lobe of the navigation signal is covered by the receiver band. Thus, $\int_{-B / 2}^{B / 2} G_{\mathrm{s}}(f) \mathrm{d} f \approx 1$. Equation (19) can then be simplified as

$$
C N 0_{\text {eff }}=\frac{a_{\mathrm{s}}^{2}}{N_{0}+T a_{1}^{2}\left|S\left(f_{\Delta}\right)\right|^{2}} .
$$

This effective $C / N_{0}$ model is the same as that in [15] and that used by Betz in research on the effects of narrowband interference with Gaussian distributions on the effective $C / N_{0}$.

\section{Case b: $\quad \eta>1$}

In this case, the carrier tracking loop tracks the filtered CWI signal. If the frequency $f_{\text {rem }}$ of the filtered CWI signal is beyond the tracking range of the PLL, the PLL will not track, but this case is not considered in this study. It is assumed in this study that the filtered CWI signal can be tracked by the PLL. Similarly, as in the case where $\eta<<1, E\{I\}$ is derived from (15):

$$
E\{I\}=a_{1}\left|S\left(f_{\Delta}-f_{\text {rem }}\right)\right|
$$

and the variance is given by

$$
\operatorname{Var}\{I(t)\}=E\left\{|I(t)|^{2}\right\}-|E\{I(t)\}|^{2} \approx \frac{N_{0}}{2 T}+\frac{a_{\mathrm{s}}^{2}}{2} .
$$

Then, the effective $C / N_{0}$ is

$$
C N 0_{\text {eff }}=\frac{a_{1}^{2}\left|S\left(f_{\Delta}-f_{\text {rem }}\right)\right|^{2}}{N_{0}+T a_{\mathrm{s}}^{2}} .
$$




\section{Case c: $\quad \eta \approx 1$}

In this case, the power levels of the filtered CWI and navigation signals are approximately equal. The carrier tracking loop may track the wrong signal or fail to track, as shown in Fig. 11. In this case, the effective $C / N_{0}$ cannot be estimated.

\section{Simulations}

Simulations were conducted using a software-defined receiver developed by Borre and Akos [18]. The DLL damping coefficient was 0.7 , the noise band was $2 \mathrm{~Hz}$, the early-late spacing was $1 / 2$ chip, the carrier tracking loop damping coefficient was 0.7 , the noise band was $25 \mathrm{~Hz}$, and all other parameter values for the receiver were chosen as in [18].

The parameters for the navigation signal were chosen as follows: the sampling frequency was $38.192 \mathrm{MHz}$, the carrier frequency was $9.548 \mathrm{MHz}$, the $\mathrm{CNR}$ was $45 \mathrm{dBHz}$, the GPS L1 C/A code PRN sequence 6 was used, the time of arrival of the navigation code $\tau_{s}$ was 0 , and the symbol rate was $1.023 \mathrm{Mcps}$. The PRN \#6 sequence was chosen to allow a comparison with the results in [15]. In [15], the effective $C / N_{0}$ was defined as follows:

$C N 0_{\mathrm{eff}}^{\mathrm{r}}=\frac{\frac{a_{\mathrm{s}}^{2}}{N_{0}} \int_{-B / 2}^{B / 2} G_{\mathrm{s}}(f) \mathrm{d} f}{\int_{-B / 2}^{B / 2} G_{\mathrm{s}}(f) \mathrm{d} f+\frac{a_{1}^{2}}{N_{0}} G_{\mathrm{s}}\left(f_{\Delta}\right)} \approx \frac{a_{\mathrm{s}}^{2}}{N_{0}+a_{1}^{2} G_{\mathrm{s}}\left(f_{\Delta}\right)}$

where the normal power spectral density of the navigation signal is defined as

$$
G_{\mathrm{s}}(f)=T \sum_{k=0}^{N-1} \sin c^{2}\left(\left(f_{\Delta}-k / T_{\mathrm{c}}\right) T\right)|C(k)|^{2} E(k)
$$

$C(k)=C_{\text {prn }}(k) / N, \quad C_{\text {prn }}(k)$ is the $N$-point fast Fourier transform (FFT) of the PRN code and $N$ is the length of the code sequence. For the GPS L1 C/A signal, $N=1023$. The signal $E(k)$ is defined as follows:

$$
E(k)=\frac{\sin ^{2}\left(\pi \frac{k}{N}\right)}{\left(\pi \frac{k}{N}\right)^{2}} .
$$

The relation between the navigation signal spectrum $S_{\mathrm{G}}(f)$ and its power spectral density $G_{\mathrm{s}}(f)$ is

$$
\begin{aligned}
\left|S_{\mathrm{G}}(f)\right| & =\sqrt{G_{\mathrm{s}}(f) / T} \\
& =\sqrt{\sum_{k=0}^{N-1} \sin c^{2}\left(\left(f_{\Delta}-k / T_{\mathrm{c}}\right) T\right)|C(k)|^{2} E(k)}
\end{aligned}
$$

Although it appears that equation (27) can be substituted into (8) as $S(f),|S(f)|$ is not equal to $\left|S_{\mathrm{G}}(f)\right|$. A plot of $|S(f)|^{2}$ and $\left|S_{\mathrm{G}}(f)\right|^{2}$ is given in Fig. 12.

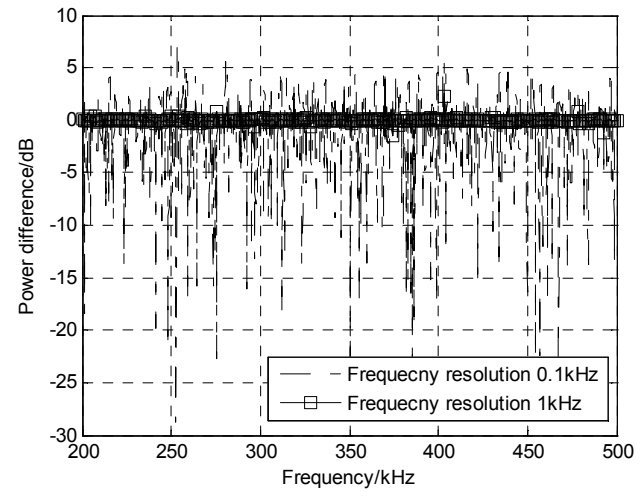

Fig. 12. Power difference between $|S(f)|^{2}$ and $\left|S_{\mathrm{G}}(f)\right|^{2}$.

Figure 12 shows the difference in power between $|S(f)|^{2}$ and $\left|S_{\mathrm{G}}(f)\right|^{2}$ as a function of the frequency, where the integration time step was $1 \mathrm{~ms}$. The power difference is insignificant at the frequencies $l / T_{\mathrm{c}}(l=0,1,2 \ldots) \mathrm{Hz}$, which can be observed from the curve for a frequency resolution of $1 \mathrm{kHz}$ shown in Fig. 12. However, the power difference may be as large as $-28 \mathrm{~dB}$, which can be observed from the curve for a frequency resolution of $0.1 \mathrm{kHz}$ shown in Fig. 12. Equation (27) can be used to estimate the spectrum at only at the frequencies $l / T_{c}$. Thus, equation (27) cannot be used to estimate $S(f)$. For this analysis, the fast Fourier transform (FFT) of the C/A code during the first $T_{\mathrm{c}}$ seconds was used to estimate $S(f)$.

It can be observed that the largest spectral line of the PRN \#6 code is at $227 \mathrm{kHz}$, and its power is approximately $-21 \mathrm{~dB}$. Therefore, when the JSR is $21 \mathrm{~dB}$, the power of the filtered CWI signal is approximately equal to that of the filtered navigation signal. The estimate of the effective $C / N_{0}$ is defined as follows:

$$
C N 0_{\text {eff }}=\frac{E\left\{\left|I_{\text {sim }}(n)\right|\right\}^{2}}{\operatorname{var}\left\{\left|I_{\text {sim }}(n)\right|\right\}}
$$

where $I_{\text {sim }}(n)$ is the real part of the output of the integratedump filter of the software-defined receiver. The effective $C / N_{0}$ update interval was $1 \mathrm{~s}$. The bit rate for the GPS C/A signal was $50 \mathrm{~Hz}$ because the duration of one data chip was $20 \mathrm{~ms}$. For an integration time $T$ of $1 \mathrm{~ms}$, there are 20 points in one data chip.

Four scenarios were simulated. The parameter values for the scenarios are shown in Tab. 1. Scenarios A and B were chosen to reveal the relation between the frequency difference and the effective $C / N_{0}$ in the presence of CWI of various power levels. Scenarios $\mathrm{C}$ and $\mathrm{D}$ were chosen to show the relation between JSR and the effective $C / N_{0}$.

It should be noted that $-226 \mathrm{kHz},-227 \mathrm{kHz}$ and $-228 \mathrm{kHz}$ are excluded. Only the CWI, the frequency of which does not coincide with the spectral lines of the navigation signal, is considered. The span of $f_{\Delta}$ in scenarios $\mathrm{A}$ and B should be the same. However, when the JSR is $50 \mathrm{dBc}, f_{\Delta}$ should be chosen to guarantee that the filtered CWI with a frequency of $f_{\text {rem }}$ can be tracked by the PLL. 


\begin{tabular}{|c|c|c|c|}
\hline Scenario & Span of $f_{\Delta}[\mathrm{kHz}]$ & $\boldsymbol{J S R}[\mathrm{dBc}]$ & Others \\
\hline A & $\begin{array}{r}-228 \sim-226 \\
\text { with step } 20 \mathrm{~Hz} \\
\end{array}$ & 8 & \multirow{4}{*}{$\begin{aligned} \tau_{s} & =0 \\
\theta_{e} & =0\end{aligned}$} \\
\hline B & $\begin{array}{c}-225.95 \sim-226.05 \\
-226.95 \sim-227.05 \\
-227.95 \sim-228.05 \\
\text { with step } 5 \mathrm{~Hz}\end{array}$ & 50 & \\
\hline C & \multirow{2}{*}{-226.95} & $\begin{array}{l}0 \sim 14 \text {; in increments } \\
\text { of } 2 \mathrm{~dB}\end{array}$ & \\
\hline D & & $\begin{array}{c}30 \sim 50 \text {; in } \\
\text { increments of } 2 \mathrm{~dB}\end{array}$ & \\
\hline
\end{tabular}

Tab. 1. Parameter values for the four scenarios.

\subsection{Scenario A}

The effective $C / N_{0}$ estimation results are shown in Fig. 13, where the horizontal axis is the frequency difference between the carrier and the CWI and the vertical axis is the effective $C / N_{0}$.

This simulation was designed to analyze the effect of the frequency on the effective $C / N_{0}$ when the power of the filtered CWI signal is less than that of the filtered navigation signal.

It can be observed that the simulation results and the analytic results obtained with the proposed model in (20) are consistent, demonstrating the accuracy of the model. The JSR was $8 \mathrm{dBc}$ in this scenario. The filtered CWI signal power was less than that of the filtered navigation signal, the PLL tracked the navigation signal, and the CWI increased the noise power after correlation, as indicated in (20). The power of the filtered CWI signal was negatively proportional to the effective $C / N_{0}$. This result is consistent with previous research such as [15], from which (24) was obtained, and explains the consistency between the effective $C / N_{0}$ computed with the proposed model and with (24). However, there were some differences in the two estimates of the effective $C / N_{0}$. These discrepancies were the result of the difference between $\left|S_{\mathrm{G}}(f)\right|^{2}$ and $|S(f)|^{2}$, which was shown in Fig. 12. The results show that the effective $C / N_{0}$ varies with the frequency difference, as indicated in (20) and (24). Because the power spectrum of the PRN code is a line spectrum, the power of the spectral lines depends on the frequency, as indicated in (8). In particular, if $f_{\Delta}=-227 \mathrm{kHz}$, the effective $C / N_{0}$ reaches a minimum because the spectral line at $227 \mathrm{kHz}$ is the strongest spectral line for PRN \#6.

\subsection{Scenario B}

The effective $C / N_{0}$ estimation results for this scenario are shown in Fig. 14, where the horizontal axis is the frequency difference between the carrier and the CWI and the vertical axis is the effective $C / N_{0}$.

The parameters for this scenario were chosen to assess the effect of the frequency difference on the effective $C / N_{0}$ when the power of filtered CWI signal is stronger than that of the filtered navigation signal.

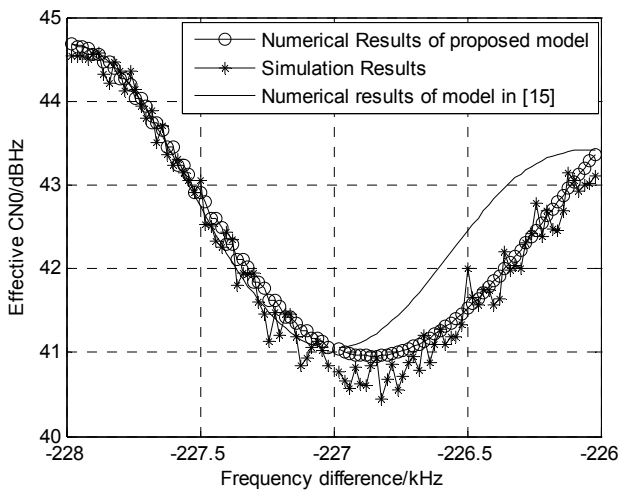

Fig. 13. Effective $C / N_{0}$ estimation results for Scenario A.

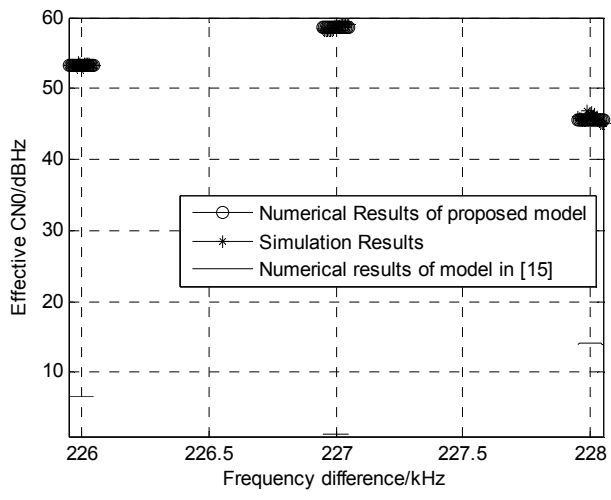

Fig. 14. Effective $C / N_{0}$ estimation results for Scenario B.

The effective $C / N_{0}$ estimates from the simulation and the proposed model were consistent, demonstrating the accuracy of (23). The effective $C / N_{0}$ varied with the frequency difference, which was explained for Scenario A. However, the results obtained with (24) were significantly different from those obtained with the proposed model. The reason for this difference is that when the power of the CWI signal is stronger than that of the navigation signal, the PLL tracks the filtered CWI signal. The CWI increases the effective carrier power, as indicated in (23). Thus, the power of the filtered CWI signal is positively proportional to the effective $C / N_{0}$. In previous studies such as [15], it was assumed that the CWI increased the noise power at the output of the correlator, but the simulation results in Fig. 14 show that this is not the case. This difference is also the reason that the effective $C / N_{0}$ computed with the proposed model reaches a maximum near $-227 \mathrm{kHz}$, whereas the effective $C / N_{0}$ computed with (24) reaches minimum. The results for this scenario reveal that the proposed model is more accurate than the model in (24).

\subsection{Scenario $\mathbf{C}$}

The results for Scenario $\mathrm{C}$ are shown in Fig. 15, where the horizontal axis is $J S R$ and the vertical axis is effective $C / N_{0}$. This scenario was designed to assess the effect of the $J S R$ on the effective $C / N_{0}$ when the power of the filtered CWI signal is less than that of the filtered navigation signal. 


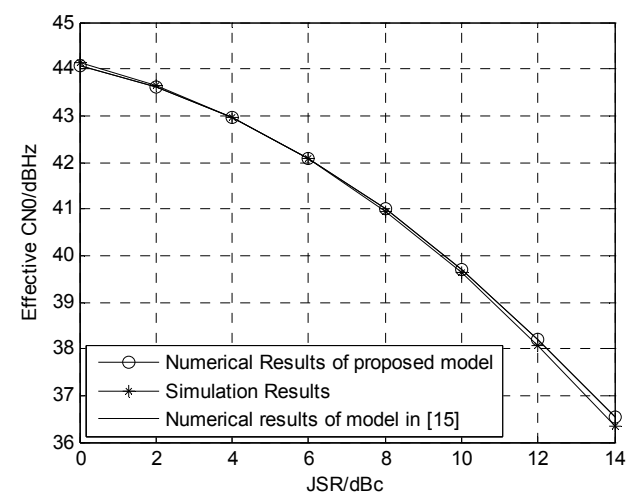

Fig. 15. Effective $C / N_{0}$ estimation results for Scenario C.

The values obtained from the simulation were consistent with those computed with the proposed model, demonstrating the accuracy of the proposed model in (20). In this scenario, the effective $C / N_{0}$ was negatively proportional to the $J S R$, as indicated in (20); the reason is the same as in Scenario A. When the power of the filtered CWI signal is less than that of the filtered navigation signal, the PLL tracks the navigation signal. The CWI increases the noise power at the output of the correlator. Thus, the value of the effective $C / N_{0}$ computed with the proposed model is approximately equal to that computed with (24).

\subsection{Scenario D}

The effective $C / N_{0}$ estimation results are shown in Fig. 16, where the horizontal axis is the JSR and the vertical axis is the effective $C / N_{0}$.

This scenario was designed to evaluate the effect of the $J S R$ on the effective $C / N_{0}$ when the power of the filtered CWI signal is stronger than that of the filtered navigation signal.

The effective $C / N_{0}$ estimates obtained from the simulation were nearly identical to those computed using (23), which demonstrates the accuracy of the proposed model. The effective $C / N_{0}$ computed with the proposed model was positively proportional to $J S R$, but the effective $C / N_{0}$ computed with (24) was negatively proportional to $J S R$, which is incorrect according to the simulation results. The reason was given in Sec. 5.2. When the power of the filtered CWI signal is stronger than that of the filtered navigation signal, the PLL tracks the filtered CWI signal. The CWI increases the effective carrier power, as indicated in (23). In contrast, the model in (24) assumes that the filtered CWI signal increases the noise power at the output of the correlator.

\subsection{Summary}

In summary, regardless of the JSR, the effective $C / N_{0}$ computed with the proposed model was consistent with the simulation results, which demonstrates the validity of the model. When the JSR is small $(\eta<<1)$, the effective $C / N_{0}$ estimated with the model in [15] was nearly identical to that computed with the proposed model, but when the JSR

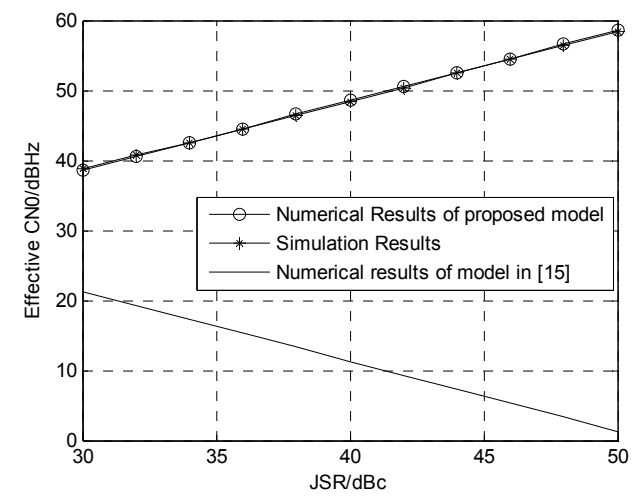

Fig. 16. Effective $C / N_{0}$ estimation results.

was large $(\eta>>1)$, the effective $C / N_{0}$ estimated with the model in [15] decreased as the JSR increased, which was the opposite of the behavior of the proposed model. The effective $C / N_{0}$ estimation model in [15] is not accurate for estimating the effective $C / N_{0}$ of receivers in the presence of CWI with a large JSR. The proposed model is more accurate.

It should be noted that an increase in the effective $C / N_{0}$ does not necessarily imply improved receiver performance. It can be observed from Scenario D that the effective $C / N_{0}$ is approximately $60 \mathrm{dBHz}$ when the $J S R$ is $50 \mathrm{dBc}$. In the presence of CWI with a $J S R$ of $50 \mathrm{dBc}$, the effective $C / N_{0}$ certainly increases. However, it can be observed that the $I$ component values in Fig. 9 are much greater than zero. Thus, the demodulation results for the navigation data are all positive in Fig. 9, which differs from the expected tracking results depicted in Fig. 6. The error bit rate of the navigation data demodulation may be $50 \%$ (the worst case). It can be concluded from this example that the increase in the effective $C / N_{0}$ does not necessarily translate to an improvement in the data demodulation performance of the receiver. The effective $C / N_{0}$ relates to receiver performance only when the effective carrier power is determined by the navigation signal and the effective noise is the result of noise plus interference. Thus, the effective $C / N_{0}$ may be misleading in evaluating receiver performance.

\section{Conclusion}

The purpose of this study was to develop a more accurate method for estimating the effective $C / N_{0}$ for GNSS receivers in the presence of CWI. The filtering of the CWI signal in the correlator was analyzed first, and then the effect of the filtered CWI signal on the carrier tracking loop was analyzed. A model for the effective $C / N_{0}$ was developed for various levels of the jam-to-signal ratio at the output of the correlator. For small values of the JSR, the proposed effective $C / N_{0}$ model was consistent with that in [15]. For large values of the JSR, the behavior of the carrier tracking loop was considered, resulting in a different effective $C / N_{0}$ model. The proposed effective $C / N_{0}$ model was validated with simulations. The proposed effec- 
tive $C / N_{0}$ model is accurate over a wider range of CWI JSR values than the model presented in [15].

An important conclusion is that the effective $C / N_{0}$ may increase in the presence of CWI. It should also be noted that receiver performance deteriorates in the presence of CWI. Therefore, the effective $C / N_{0}$ may not be accurate in evaluating receiver performance in certain cases. The effective $C / N_{0}$ relates to receiver performance only when the effective carrier power results from the navigation signal and the effective noise results from the noise plus interference.

Several problems remain. As mentioned previously, the effective $C / N_{0}$ may not accurately reflect receiver tracking performance and data demodulation performance in the presence of CWI. An appropriate method for evaluating receiver performance in the presence of CWI remains to be developed.

\section{Acknowledgments}

The authors would like to express their gratitude to Dr. Zhuang, a respectable and resourceful scholar, for his guidance and to the instructors and colleagues at the College of Electronic Science and Engineering, National University of Defense Technology, for their help and support.

\section{References}

[1] BETZ, J. W. Effect of partial-band interference on receiver estimation of CN0 theory. Technical Report, the MITRE Corporation, 2001, p. 716-723.

[2] BETZ, J. W. Effect of narrowband interference on GPS code tracking accuracy. In Proceedings of 2000 National Technical Meeting of the Institute of Navigation. Anaheim (CA, USA), January 2000, p. 16-27.

[3] BETZ, J. W., KOLODZIEJSKI, K. R. Generalized theory of code tracking with an early-late discriminator part 1: Lower bound and coherent processing. IEEE Transactions on Aerospace and Electronic Systems, 2009, vol. 45, no. 4, p. 1538-1550. DOI: 10.1109/TAES.2009.5310316

[4] BETZ, J. W., KOLODZIEJSKI, K. R. Generalized theory of code tracking with an early-late discriminator part 2: Non-coherent processing and numerical results. IEEE Transactions on Aerospace and Electronic Systems, 2009, vol. 45, no. 4, p. 1551-1564. DOI: 10.1109/TAES.2009.5310317

[5] KAPLAN, E. D., HEGARTY, C. J. Understanding GPS: Principles and Applications. $2^{\text {nd }}$ ed. London: The Artech House, 2006. ISBN: 1580538940

[6] MOTElla, B., SAVASTA, S., MARGARIA, D., et al. Method for assessing the interference impact on GNSS receivers. IEEE Transactions on Aerospace and Electronic Systems, 2011, vol. 47, no. 2, p. 1416-1432. DOI: 10.1109/TAES.2011.5751267

[7] ZHANG, J., LOHAN, E. S. Effect of narrowband interference on Galileo E1 signal receiver performance. International Journal of Navigation and Observation, 2011, vol. 2011, p. 1-10. DOI: $10.1155 / 2011 / 959871$
[8] BEK, M. K., SHAHEEN, E. M., ELGAMEL, S. A. Mathematical analyses of pulse interference signal on post-correlation carrier-tonoise ratio for the global positioning system receivers. IET Radar, Sonar and Navigation, 2015, vol. 9, no. 3, p. 266-275. DOI: 10.1049/iet-rsn.2014.0155

[9] OJEDA, O. A. Y., GRAJAL, J., LOPEZ-RISUENO, G. Analytical performance of GNSS receivers using interference mitigation techniques. IEEE Transactions on Aerospace and Electronic Systems, 2013, vol. 49, no. 2, p. 885-906. DOI: 10.1109/TAES.2013.6494387

[10] ZHANG, T., ZHANG, X., LU, M. Effect of frequency domain anti-jamming filter on satellite navigation signal tracking performance. In Proceedings of 4th China Satellite Navigation Conference. The Wuhan, May 2013, p. 507-516.

[11] MUSUMECI, L., SAMSON, J., DOVIS, F. Performance assessment of pulse blanking mitigation in presence of multiple distance measuring equipment/tactical air navigation interference on global navigation satellite systems signals. IET Radar, Sonar and Navigation, 2014, vol. 8, no. 6, p. 647-657. DOI: 10.1049/ietrsn.2013.0198

[12] BORIO, D. GNSS acquisition in the presence of continuous wave interference. IEEE Transactions on Aerospace and Electronic Systems, 2010, vol. 46, no. 1, p. 47-60. DOI: 10.1109/TAES.2010.5417147

[13] LIU, Y., RAN, Y., KE, T., et al. Code tracking performance analysis of GNSS signal in the presence of CW interference. Signal Processing, 2011, vol. 91, no. 4, p. 970-987. DOI: 10.1016/j.sigpro.2010.09.022

[14] BALAEI, A. T., DEMPSTER, A. G., PRESTI, L. L. Characterization of the effects of $\mathrm{CW}$ and pulse $\mathrm{CW}$ interference on the GPS signal quality. IEEE Transactions on Aerospace and Electronic Systems, 2009, vol. 45, no. 4, p. 1418-1431. DOI: 10.1109/TAES.2009.5310308

[15] JANG, J., PAONNI, M., EISSFELLER, B. CW interference effects on tracking performance of GNSS receivers. IEEE Transactions on Aerospace and Electronic Systems, 2012, vol. 48, no. 1, p. 243-258. DOI: 10.1109/TAES.2012.6129633

[16] POtTer, B. J., SHAlberG, K., GRABOWSKI, J. Personal privacy device interference in the WAAS. In Proceedings of the $25^{\text {th }}$ International Technical Meeting of the Satellite Division of the Institute of Navigation. Nashville (TN, USA), September 2012, p. 2868-2874.

[17] MARCUS, M. J. Growing consumer interest in jamming: spectrum policy implications. IEEE Wireless Communications, 2014, vol. 21 , no. 1, p. 4. DOI: 10.1109/MWC.2014.6757888

[18] BORRE, K., AKOS, D. M., BERTELSEN, N., et al. A soft-defined GPS and Galileo Receiver. 1st ed. Boston: The Birkhauser, 2007. ISBN: 9780817645403

\section{About the Authors ...}

Jian LI was born in 1988. He is a Ph.D. candidate in the Information and Communication Engineering Department at the National University of Defense Technology (NUDT) in China. He received his M.Sc. from the Aviation University of the Chinese Air Force in 2012. His research interests include radio frequency interference monitoring and anti-interference techniques for GNSS.

Junwei NIE was born in 1983. He is a lecturer at the National University of Defense Technology, where he received his Ph.D. degree from the School of Electronic Science and Engineering in 2012. His research interests 
include radio frequency interference and anti-interference techniques in GNSS.

Baiyu LI was born in 1983. He is a lecturer at the National University of Defense Technology, where he received his Ph.D. degree from the School of Electronic Science and Engineering in 2012. His research interests include radio frequency front-end techniques for GNSS.

Feixue WANG (corresponding author) was born in 1965. $\mathrm{He}$ is a Full Professor in Electronic Science and Engineering at the National University of Defense Technology. He received his Ph.D. degree in 1998. He has researched and developed navigation systems for more than 18 years. His research interests include navigation signal design, signal processing, data processing, and anti-jamming methods for GNSS.

\section{Appendix A}

The early component of the signal at the output of the DLL code correlator is defined as follows:

$$
\begin{aligned}
E & =\left|r\left(\theta, \tau+\frac{\Delta}{2}\right)\right|^{2} \\
& =\left|r_{\mathrm{ss}}\left(\theta, \tau+\frac{\Delta}{2}\right)+r_{\mathrm{ls}}\left(\theta, \tau+\frac{\Delta}{2}\right)+r_{\mathrm{ws}}\left(\theta, \tau+\frac{\Delta}{2}\right)\right|^{2} \\
& =\left|r_{\mathrm{ss}}\left(\theta, \tau+\frac{\Delta}{2}\right)\right|^{2}+\left|r_{\mathrm{ls}}\left(\theta, \tau+\frac{\Delta}{2}\right)\right|^{2}+\left|r_{\mathrm{ws}}\left(\theta, \tau+\frac{\Delta}{2}\right)\right|^{2} \\
& +2 \operatorname{Re}\left\{r_{\mathrm{ss}}\left(\theta, \tau+\frac{\Delta}{2}\right) r_{\mathrm{ls}}^{*}\left(\theta, \tau+\frac{\Delta}{2}\right)\right. \\
& +r_{\mathrm{ss}}\left(\theta, \tau+\frac{\Delta}{2}\right) r_{\mathrm{ws}}^{*}\left(\theta, \tau+\frac{\Delta}{2}\right) \\
& \left.+r_{\mathrm{ls}}\left(\theta, \tau+\frac{\Delta}{2}\right) r_{\mathrm{ws}}^{*}\left(\theta, \tau+\frac{\Delta}{2}\right)\right\}
\end{aligned}
$$

where $r_{\mathrm{ss}}, r_{\mathrm{ls}}$ and $r_{\mathrm{ws}}$ were defined in (4). The late component of the signal at the output of the DLL code correlator is defined as follows:

$$
\begin{aligned}
L= & \left|r_{\mathrm{ss}}\left(\theta, \tau-\frac{\Delta}{2}\right)\right|^{2}+\left|r_{\mathrm{ls}}\left(\theta, \tau-\frac{\Delta}{2}\right)\right|^{2}+\left|r_{\mathrm{ws}}\left(\theta, \tau-\frac{\Delta}{2}\right)\right|^{2} \\
& +2 \operatorname{Re}\left\{r_{\mathrm{ss}}\left(\theta, \tau-\frac{\Delta}{2}\right) r_{\mathrm{ls}}^{*}\left(\theta, \tau-\frac{\Delta}{2}\right)\right. \\
& +r_{\mathrm{ss}}\left(\theta, \tau-\frac{\Delta}{2}\right) r_{\mathrm{ws}}^{*}\left(\theta, \tau-\frac{\Delta}{2}\right) \\
& \left.+r_{\mathrm{ls}}\left(\theta, \tau-\frac{\Delta}{2}\right) r_{\mathrm{ws}}^{*}\left(\theta, \tau-\frac{\Delta}{2}\right)\right\} .
\end{aligned}
$$

The noise is assumed to be Gaussian. The error signal at the output of code correlator is

$$
\begin{aligned}
& E\{E-L\} \\
= & \left|r_{\mathrm{ss}}\left(\theta, \tau+\frac{\Delta}{2}\right)\right|^{2}-\left|r_{\mathrm{ss}}\left(\theta, \tau-\frac{\Delta}{2}\right)\right|^{2} \\
& +\left|r_{\mathrm{ls}}\left(\theta, \tau+\frac{\Delta}{2}\right)\right|^{2}-\left|r_{\mathrm{ls}}\left(\theta, \tau-\frac{\Delta}{2}\right)\right|^{2} \\
& +2 \operatorname{Re}\left\{r_{\mathrm{ss}}\left(\theta, \tau+\frac{\Delta}{2}\right) r_{\mathrm{ls}}^{*}\left(\theta, \tau+\frac{\Delta}{2}\right)\right. \\
& \left.-r_{\mathrm{ss}}\left(\theta, \tau-\frac{\Delta}{2}\right) r_{\mathrm{ls}}^{*}\left(\theta, \tau-\frac{\Delta}{2}\right)\right\} .
\end{aligned}
$$

\section{Case a: $\quad \eta<<1$}

When the PLL tracks the navigation signal, the filtered signal can be expressed as follows:

$$
\begin{aligned}
& r_{\mathrm{ss}}\left(\theta, \tau+\frac{\Delta}{2}\right) \\
= & \frac{1}{T} \int_{-T / 2}^{T / 2} \exp (j \theta) d(t) s(t) s_{0}^{*}(t-\tau-\Delta / 2) \mathrm{d} t \\
= & \frac{a_{\mathrm{s}}}{T} d(T) \exp (j \theta) \int_{-B / 2}^{B / 2} \exp [j 2 \pi f(\tau+\Delta / 2)] s(f) \\
& \int_{-\frac{T}{2}-\tau-\Delta / 2}^{\frac{T}{2}-\tau-\Delta / 2} s_{0}^{*}(u) \exp (j 2 \pi f u) \mathrm{d} u \mathrm{~d} f \\
\approx & a_{\mathrm{s}} d(T) \exp (j \theta) \int_{-B / 2}^{B / 2} \exp [j 2 \pi f(\tau+\Delta / 2)] G_{\mathrm{s}}(f) \mathrm{d} f \\
= & a_{\mathrm{s}} d(T) \exp (j \theta) R(\tau+\Delta / 2)
\end{aligned}
$$

where $R_{\Delta / 2}=R(\tau+\Delta / 2)$ and $R_{-\Delta / 2}=R(\tau-\Delta / 2)$. Thus, the difference in the filtered signal terms for early and late branch is

$$
\begin{aligned}
& \left|r_{\mathrm{ss}}\left(\theta, \tau+\frac{\Delta}{2}\right)\right|^{2}-\left|r_{\mathrm{ss}}\left(\theta, \tau-\frac{\Delta}{2}\right)\right|^{2} \\
& =a_{\mathrm{s}}^{2} R_{\Delta / 2}^{2}-a_{\mathrm{s}}^{2} R_{-\Delta / 2}^{2} .
\end{aligned}
$$

An expression for the filtered interference can be derived from (5):

$$
\begin{aligned}
& r_{\mathrm{ls}}\left(\theta, \tau+\frac{\Delta}{2}\right) \\
& =a_{1}\left|S_{\tau+\Delta / 2}\left(f_{\Delta}\right)\right| \exp j\left(2 \pi f_{\mathrm{rem}} U-\phi+\beta+\pi f_{\Delta} \Delta\right) .
\end{aligned}
$$

The difference in the interference is

$$
\begin{aligned}
& \left|r_{\text {ls }}\left(\theta, \tau+\frac{\Delta}{2}\right)\right|^{2}-\left|r_{\text {ls }}\left(\theta, \tau-\frac{\Delta}{2}\right)\right|^{2} \\
& =a_{1}^{2}\left|S_{\tau+\Delta / 2}\left(f_{\Delta}\right)\right|^{2}-a_{1}^{2}\left|S_{\tau-\Delta / 2}\left(f_{\Delta}\right)\right|^{2} \\
& =0 .
\end{aligned}
$$

A cross term between the signal and the interference can be defined as follows: 


$$
\begin{aligned}
& r_{\mathrm{ss}}\left(\theta, \tau+\frac{\Delta}{2}\right) r_{\mathrm{ls}}^{*}\left(\theta, \tau+\frac{\Delta}{2}\right) \\
& =a_{\mathrm{s}} d(T) \exp (j \theta) R(\tau+\Delta / 2) \\
& a_{1}\left|S\left(f_{\Delta}\right)\right| \exp \left[-j\left(2 \pi f_{\mathrm{rem}} U-\phi+\beta+\pi f_{\Delta} \Delta\right)\right] \\
& =a_{\mathrm{s}} a_{1} d(T) R_{\Delta / 2}\left|S\left(f_{\Delta}\right)\right| \\
& \exp \left[-j\left(2 \pi f_{\mathrm{rem}} U-\phi+\beta+\pi f_{\Delta} \Delta-\theta\right)\right] .
\end{aligned}
$$

Thus, the difference in the cross terms is

$$
\begin{gathered}
\operatorname{Re}\left\{\begin{array}{l}
r_{\mathrm{ss}}\left(\theta, \tau+\frac{\Delta}{2}\right) r_{\mathrm{ls}}^{*}\left(\theta, \tau+\frac{\Delta}{2}\right) \\
-r_{\mathrm{ss}}\left(\theta, \tau-\frac{\Delta}{2}\right) r_{\mathrm{ls}}^{*}\left(\theta, \tau-\frac{\Delta}{2}\right)
\end{array}\right\} \\
=\operatorname{Re}\left\{\begin{array}{l}
a_{\mathrm{s}} a_{1} d(T)\left|S\left(f_{\Delta}\right)\right| \exp \left[-j\left(2 \pi f_{\mathrm{rem}} U-\phi+\beta-\theta\right)\right] \\
\left(R_{\Delta / 2} \exp \left(-j \pi f_{\Delta} \Delta\right)-R_{-\Delta / 2} \exp \left(j \pi f_{\Delta} \Delta\right)\right)
\end{array}\right\} .
\end{gathered}
$$

Then,

$$
E\left\{\operatorname{Re}\left\{\begin{array}{l}
r_{\mathrm{ss}}\left(\theta, \tau+\frac{\Delta}{2}\right) r_{\mathrm{ls}}^{*}\left(\theta, \tau+\frac{\Delta}{2}\right) \\
-r_{\mathrm{ss}}\left(\theta, \tau-\frac{\Delta}{2}\right) r_{\mathrm{ls}}^{*}\left(\theta, \tau-\frac{\Delta}{2}\right)
\end{array}\right\}\right\}=0
$$

Thus, the signal at the output of code correlator using NELP is

$$
E\{E-L\}=a_{\mathrm{s}}^{2} R_{\Delta / 2}^{2}-a_{\mathrm{s}}^{2} R_{-\Delta / 2}^{2}
$$

It can be concluded from (39) that the code tracking is unbiased; that is, $\tau=0$.

\section{Case b: $\quad \eta>>1$}

When the PLL tracks the filtered CWI signal, the $f_{\text {rem }}$ frequency component will be removed from the CWI. Thus, the frequency of the replica carrier is $f_{\mathrm{c}}+f_{\mathrm{d}}+f_{\text {rem }}$. Thus, the filtered signal term can be expressed as follows:

$$
\begin{aligned}
& r_{\mathrm{ss}}\left(\theta, \tau+\frac{\Delta}{2}\right) \\
= & \frac{1}{T} \int_{-T / 2}^{T / 2} d(t) \exp (j \theta) s(t) s_{0}^{*}\left(t-\tau-\frac{\Delta}{2}\right) \mathrm{d} t \\
= & \frac{a_{\mathrm{s}}}{T} d(T) \exp \left[j\left(\theta+\theta_{\mathrm{s}}-\theta_{l}\right)\right] \\
& \int_{-T / 2}^{T / 2} c\left(t-\tau_{\mathrm{s}}\right) c\left(t-\tau_{\mathrm{s}}-\tau-\frac{\Delta}{2}\right) \exp \left(-j 2 \pi f_{\mathrm{rem}} t\right) \mathrm{d} t \\
= & a_{\mathrm{s}} d(T)\left|S_{\tau+\Delta / 2}^{\prime}\left(f_{\mathrm{rem}}\right)\right| \exp \left[j\left(2 \pi f_{\text {rem }} U+\theta_{\text {ss }}\right)\right] .
\end{aligned}
$$

where $S_{\tau+\Delta / 2}^{\prime}(f)$ is the spectrum of the PRN code, $c_{\tau+\Delta / 2}^{\prime}(t)=c\left(t-\tau_{\mathrm{s}}\right) c\left(t-\tau_{\mathrm{s}}-\tau-\Delta / 2\right)$ and $\theta_{\mathrm{ss}}$ is the initial phase of the filtered signal. Thus, the filtered signal can be expressed as follows:

$$
\begin{aligned}
& \left|r_{\mathrm{ss}}\left(\theta, \tau+\frac{\Delta}{2}\right)\right|^{2}-\left|r_{\mathrm{ss}}\left(\theta, \tau-\frac{\Delta}{2}\right)\right|^{2} \\
& =a_{\mathrm{s}}^{2}\left|S_{\tau+\Delta / 2}^{\prime}\left(f_{\text {rem }}\right)\right|^{2}-a_{\mathrm{s}}^{2}\left|S_{\tau+\Delta / 2}^{\prime}\left(f_{\text {rem }}\right)\right|^{2} \\
& \approx 0
\end{aligned}
$$

Because the filtered CWI signal is tracked by the PLL, the interference term becomes a constant. The interference can be expressed as follows:

$$
\begin{aligned}
& r_{\mathrm{ls}}\left(\theta, \tau+\frac{\Delta}{2}\right) \\
& =\frac{1}{T} \int_{-T / 2}^{T / 2} \exp (j \theta) d(t) l(t) s_{0}^{*}\left(t-\tau-\frac{\Delta}{2}\right) \mathrm{d} t \\
& =\frac{a_{1}}{T} d(T) \int_{-T / 2}^{T / 2} c\left(t-\tau_{\mathrm{s}}-\tau-\frac{\Delta}{2}\right) \\
& \exp j\left(\theta+\theta_{l}-\theta_{s}\right) \exp \left[j 2 \pi\left(f_{\Delta}-f_{\text {rem }}\right) t\right] \mathrm{d} t \\
& =a_{1} d(T)\left|S_{\tau+\Delta / 2}\left(f_{\Delta}-f_{\text {rem }}\right)\right| \exp \left(j \theta_{\mathrm{ls}}\right) .
\end{aligned}
$$

The difference in the interference is

$$
\begin{aligned}
& \left|r_{\mathrm{ls}}\left(\theta, \tau+\frac{\Delta}{2}\right)\right|^{2}-\left|r_{\mathrm{ls}}\left(\theta, \tau-\frac{\Delta}{2}\right)\right|^{2} \\
& =a_{1}^{2}\left|S_{\tau+\Delta / 2}\left(f_{\Delta}-f_{\text {rem }}\right)\right|^{2}-a_{1}^{2}\left|S_{\tau-\Delta / 2}\left(f_{\Delta}-f_{\text {rem }}\right)\right|^{2} \\
& =0
\end{aligned}
$$

A cross term between the signal and the interference can be defined as follows:

$$
\begin{aligned}
& r_{\mathrm{ss}}\left(\theta, \tau+\frac{\Delta}{2}\right) r_{\mathrm{ls}}^{*}\left(\theta, \tau+\frac{\Delta}{2}\right) \\
& =a_{\mathrm{s}} d(T)\left|S_{\tau+\Delta / 2}^{\prime}\left(f_{\Delta}\right)\right| \exp \left[j\left(2 \pi f_{\mathrm{rem}} U+\theta_{\mathrm{ss}}\right)\right] \\
& a_{1}\left|S_{\tau+\Delta / 2}\left(f_{\Delta}-f_{\mathrm{rem}}\right)\right| \exp \left(-j \theta_{\mathrm{ls}}\right) \\
& =a_{\mathrm{s}} a_{1} d(T)\left|S_{\tau+\Delta / 2}^{\prime}\left(f_{\Delta}\right)\right| \\
& \left|S_{\tau+\Delta / 2}\left(f_{\Delta}-f_{\mathrm{rem}}\right)\right| \exp \left[j\left(2 \pi f_{\mathrm{rem}} U+\theta_{\mathrm{ss}}-\theta_{\mathrm{ls}}\right)\right] .
\end{aligned}
$$

The difference in the cross terms is

$$
E\left\{\operatorname{Re}\left\{\begin{array}{l}
r_{\mathrm{ss}}\left(\theta, \tau+\frac{\Delta}{2}\right) r_{\mathrm{ls}}^{*}\left(\theta, \tau+\frac{\Delta}{2}\right) \\
-r_{\mathrm{ss}}\left(\theta, \tau-\frac{\Delta}{2}\right) r_{\mathrm{ls}}^{*}\left(\theta, \tau-\frac{\Delta}{2}\right)
\end{array}\right\}\right\}=0 .
$$

Thus, the signal at the output of the code correlator using NELP is

$$
E\{E-L\}=0
$$

It can be concluded from (46) that the code tracking error is unchanged.

In summary, CWI will not change the code tracking performance. 